\title{
A Case Study Exploring the Influence of a Gardening Therapy Group on Well-Being
}

\author{
Joyce, J
}

http://hdl.handle.net/10026.1/5338

\subsection{0/0164212X.2015.1111184}

Occupational Therapy in Mental Health

Taylor \& Francis (Routledge)

All content in PEARL is protected by copyright law. Author manuscripts are made available in accordance with publisher policies. Please cite only the published version using the details provided on the item record or document. In the absence of an open licence (e.g. Creative Commons), permissions for further reuse of content should be sought from the publisher or author. 
This was published in

Joyce, J., \& Warren, A. (2016) 'A Case Study Exploring the Influence of a Gardening Therapy group on WellBeing'. Occupational Therapy in Mental Health, 32(2), 203-215

And can be found at

DOI: 10.1080/0164212X.2015.1111184

A Case Study Exploring the Influence of a Gardening Therapy Group on Well-being

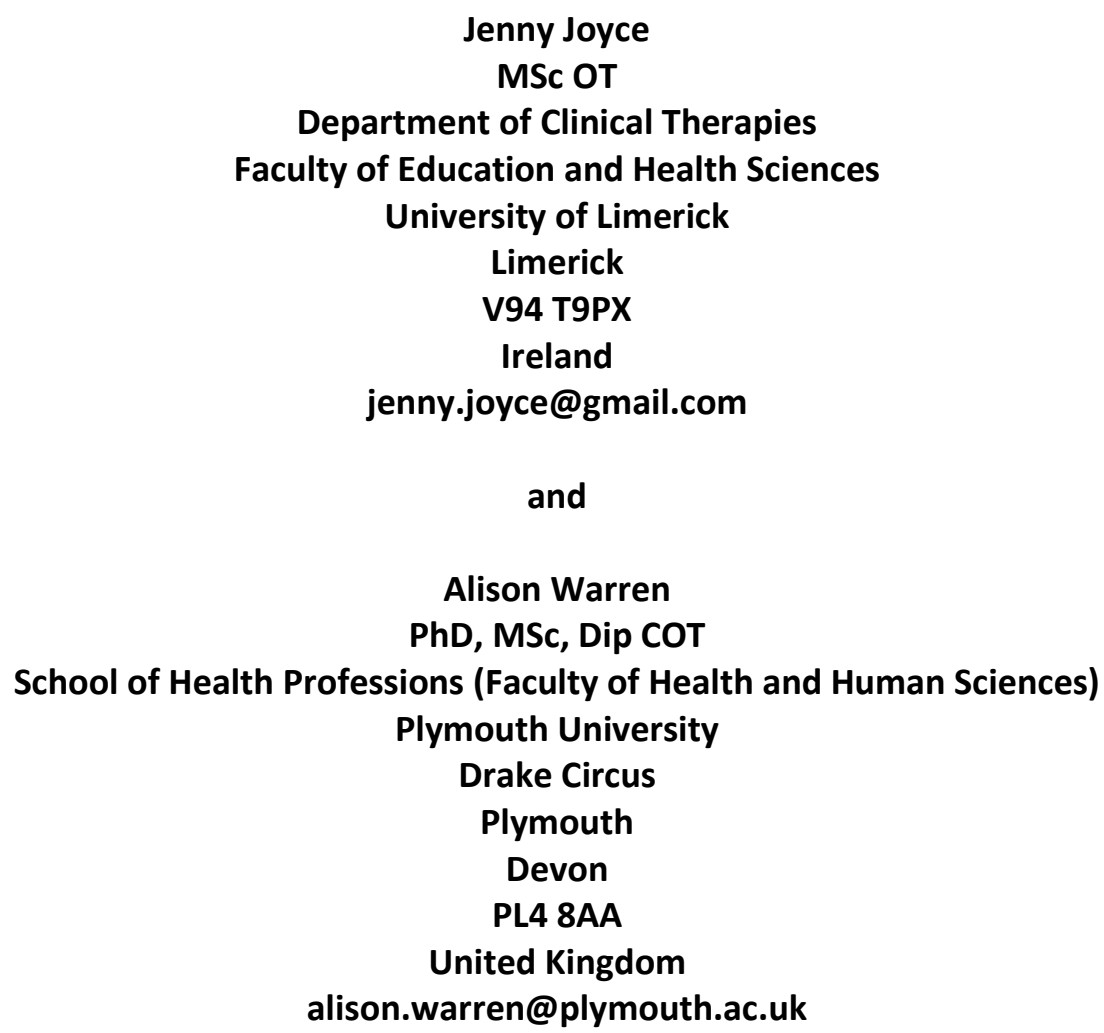

Address correspondence to: Jenny Joyce: jenny.joyce@gmail.com 


\begin{abstract}
In a climate of improving health care quality and understanding, this study explored how participation in a gardening group, facilitated through a mental health service in Ireland, influenced well-being. A case study used semi-structured interviews and participant observation to explore explicit and tacit aspects of group participation. Thematic analysis clarified that gardening group participation had a positive influence on well-being for all gardeners and group facilitators. This was through promoting coping, facilitating change, and developing skills. The setting, site ethos and tasks were important in assisting people to make lifestyle changes and to develop skills in order to experience well-being.
\end{abstract}

KEYWORDS. Occupational therapy, gardening, horticulture therapy, social, group case study 


\section{Introduction}

There is a need to further support the benefits of mental health occupational therapy in an Irish setting (O'Connell \& McKay, 2010). According to International findings, mental health occupational therapists struggle to produce and disseminate their distinct influence on health care (Casteleijn \& Graham, 2012) and as such, more evidence is required on the benefits of occupational therapy for people with mental ill-health (Mee, Sumsion, \& Craik, 2004). This is especially true of gardening interventions facilitated by occupational therapists, as distinct from therapeutic gardening groups facilitated by a registered and certified horticultural therapist. While horticultural therapists exclusively use the horticultural milieu as their therapeutic tool (AHTA, 2015), occupational therapists facilitate the use of a person's meaningful occupations as a tool for well-being (Wilcock, 2006). There is a growing body of evidence to support the use of both Horticultural Therapy, and gardening groups facilitated in an occupational therapy setting, but further research is needed to explore the processes of such experiences (Fieldhouse \& Sempik, 2007; Newton, 2007; Sempik, Rickhuss \& Beeston, 2014).

The impetus for this study occurred when occupational therapists in a mental health service noted anecdotally the positive influence of participation in the service's weekly allotment gardening group on members' well-being. Allotment is a British term, defined as "A plot of land rented by an individual for growing vegetables or flowers" (Oxford Dictionaries, 2015). The gardening group had been established to assist participants in gaining skills and to promote their recovery from mental ill health. A body of international evidence for the general use of a gardening group as a therapeutic medium, as well as a perceived fit with the interests of the people using the service, made the group a clear 
choice for the service to implement. Furthermore, the therapists wished to be able to describe and convey the influence of participation on the members' well-being. As such, this research collaboration aimed to explore the influence of participation in the weekly gardening group on the well-being of individuals involved.

\section{Literature Review}

A literature search was conducted using the terms gardening, allotments, horticulture, mental health, well-being and outdoor occupation within the CINAHL, Medline, PsychINFO, AMED databases and Google Scholar to seek papers related to well-being, occupational therapy, and horticulture from the last twenty-five years. The literature relating to the concept of well-being brought to light themes apropos of pursuing one's own personal aspirations and of being capable of engaging in daily life (self-determination). Other facets of well-being in the literature identified were happiness, a sense of growth, a transcendence of the limitations of the body, space, time and circumstances, social and mental health, belonging, and a feeling of being at peace with one's self and others, often through meaningful occupation (Johnson \& Schmidt, 1983; Wilcock, 2006). However, there is a growing emphasis on highlighting the importance of culture and community in mental health and well-being (Iwama, 2003). As such, it should be observed that this study was completed in an Irish context with many inherent values based on Western assumptions.

The physical environment also plays a role in well-being. In studies on green exercise, it was found that viewing images of nature can improve the beneficial effect of exercise on well-being, even if indoors, while engaging in exercise in nature was seen to immediately improve the same benefits (Pretty, Peacock, Hine, Sellens, South \& Griffin, 2007; Pretty, Peacock, Sellens \& Griffin, 2005). In terms of well-being and gardening, Frances (2006) reported that outdoor activities in general are feasible therapeutic media for people with enduring mental health problems. Gonzalez, Hartig, Patil, Martinsen, and Kirkevold (2010) 
highlighted how cognitive engagement with therapeutic horticulture was associated with a reduction in symptoms associated with depression.

Moreover, a critical review on the use of gardening as an occupation has underlined the multifaceted potential of gardening to promote well-being through not only the environmental aspects outlined above, but through other, more complex, social aspects (York \& Wiseman, 2012). Fieldhouse's (2003) study, using gardener focus groups, highlighted gardening as a potentially benign, stigma free and encouraging place that held a wide range of benefits pertaining to the interaction of social, cognitive and spiritual aspects of wellbeing. A similar interplay was observed between community gardens, the promotion of physical exercise, and psychological well-being (Stein, 2008).

As previous literature highlights the importance of the environment in such interventions (Diamant \& Waterhouse, 2010; Parkinson, Lowe, \& Vecsey, 2011) and as this study considered community well-being in a therapeutic setting it was prudent to address literature on optimal levels of integration for such activities. Bates, Gee, Klingel, and Lippman (2006) suggested that segregated therapeutic groups may not support inclusion and coping in the community, while research by Nagle, Cook, and Polatajko (2002) suggested an individually graded approach to integration is necessary to limit a person's perceived risks of a relapse. In relation to addressing this issue, Diamant and Waterhouse (2010) reported on the importance of the just right, safe, graded environment in obtaining the benefits from engaging in social gardening.

In summation, a review of available literature revealed a need for further research focus on therapeutic or social aspects of gardening, and that evidence to date for the benefits of gardening as a medium for mental health occupational therapy has been anecdotal, unreliable or has suffered from ill-defined study designs (Sempik, Aldridge, \& Becker, 2005; York \& Wiseman, 2012). 
Furthermore the literature highlights the importance of conducting additional qualitative studies of occupational therapist-led allotment groups to unwrap and explore the possible social dynamics involved (Newton, 2007). Therefore this study sought to explore the question "How does participation in an allotment [gardening] group, facilitated through a mental health occupational therapy service, influence well-being?"

\section{Methodology}

A qualitative case study methodology was utilized to allow the deep exploration of the rich and complex emic (insider) perspective of the people involved in the group and their voices within it (Yin, 2009). The research paradigm aligns with that of Critical Theory in an attempt to challenge the traditional notions of mental health treatments and promote research participation collaboration from those using or working in mental health service (Angrosino, 2007). These methodological considerations facilitated the investigation of the experience of people in a specific allotment group with implicit rules through collation of information from a range of methods/perspectives.

The group was facilitated in the community, away from the hospital setting, on two sites; one with an open garden space and one with a poly-tunnel or cover. The interviews were completed in a familiar private office in a mental health service, with participant observation at the nearby community allotment site. Research methods included semi-structured interviews, participant observation with the completion of a reflective journal by the primary researcher. Individual interviews were conducted with facilitators and gardeners in the office setting to address issues of confidentiality or speaking in front of others while allowing exploration of each participant's views and experiences related to their individual gardening participation experience (MacDougall \& Fudge 2001).

The researchers were not members of staff within the mental health service. Participant observation was employed on the site where gardening is used as a medium for occupational therapy. The aim of this method was to explore tacit factors pertaining to the 
group, regarding which participants may have not been aware of or able to articulate (Depoy \& Gitlin 2005; Mason, 2002). This method is compatible in triangulation with the other methods used, and is considered to be less biased, invasive and scrutinizing than direct observation (Angrosino, 2007).

For this study, purposive sampling using a gatekeeper within the mental health service was employed. Potential participants received an information sheet and willing participants contacted the researcher to arrange an interview time at their convenience. A pilot interview was conducted which resulted in simplification of the language on the interview sheet and a reordering of questions to achieve a better flow. Interview questions included: tell me about your involvement with the gardening group? How do you think you have benefitted from taking part in the group? Has the gardening group brought you any challenges and what do you think is the most important thing about setting up a gardening group? Data analyzed for this study included transcripts from participant interviews, along with researcher field-notes from participant observation.

Thematic analysis consisted of the first author reading all data twice, making note of rudimentary codes by hand, exploring initial themes (Braun \& Clarke, 2006) and concept mapping with NVivo qualitative data analysis software (QSR International, 2002). Themes were then reviewed through discussion and visual analysis with the second author. This reconfigured and recontextualized themes to be more flowing and valid where the master themes were synthesized from seven to two (figure 1). Themes were then finalized to include extracts corresponding to emergent themes from each data source. This enabled the researcher to report quotes that reflected overall accounts reported by participants.

This study aimed to address the four markers of trustworthiness in qualitative research as outlined by Krefting (2001): credibility, transferability, dependability and confirmability. In terms of credibility, the study aimed to represent a recognizable picture of the people's experiences through capturing personal experience in interviews and through attending the 
group over a prolonged period of four visits. This was supported through a member check, with a 50\% response rate (two gardeners and one facilitator) all of which agreed with the findings. The primary researcher also kept a reflective diary that was discussed in research supervision to maintain the focus on the participants' experience. Transferability was addressed through comparing emergent themes with those reflected in previous research, and through giving a rich, thick and deep report of data in order to allow others to assess goodness of fit with other contexts. Strategies to address dependability and confirmability involved giving a clear account of the research conducted through review and audit by the second author.

\section{Research ethics:}

Ethical approval for this study and its publication was obtained from the Health Service Executive in the relevant county in Ireland. As participant observation was a major source of data collection and contextualization for the study it was vital to address the documented ethical issues associated with the method. Issues of perceived duplicity and role blurring for the researcher between the emic and etic perspectives (Mason, 2002) were addressed through overt participant observation by which all gardeners and facilitators were aware of the information and experience gathering role of the researcher. Transparency was further assisted by the researcher completing reflective journal entries and field notes after sessions to explicate assumptions and observations. To ensure the least interruption to the group, the participant observation sessions took place only as part of the officially scheduled weekly gardening times.

Potential power issues were involved in the dual role of the current group facilitator, as both a participant and the gatekeeper to the study. These issues were addressed by the researcher seeking renewed consent from all participants at every step of the process, and ensuring that all participants were empowered to withdraw from the study without repercussion on group attendance. Any potential issues of identifying a person's 
contribution have been lessened through the use of individual interviews and pseudonyms, along with thematic reporting and through storage of information in line with local data protection guidelines.

\section{Findings}

\section{Participants}

This study had six participants; three men and three women, with three gardening group facilitators (two occupational therapists and one social worker) and three gardeners who were local mental health service users living in the community.

\section{Themes}

From thematic analysis of interview transcripts and participant observation field notes two master themes emerged with six sub themes, which are outlined in Figure 1.

\section{Figure 1: Master Themes and Sub Themes here}

The two master themes are "influences on well-being" and "mechanisms of well-being." Influences on well-being emerged regarding 1) the group setting, 2) the tasks involved, and 3) the ethos of the group. These influences were revealed as contributing to well-being through participation by three mechanisms of well-being: 1) promoting coping, 2) facilitating change, and 3) providing opportunities for skill development (see figure 1). When discussing the influence of the gardening group on well-being, all gardeners reported agreement regarding the influence of participation on well-being, for example: "There's contentment in it ...there's happiness in the success."- David, gardener. Two out of three facilitators referred specifically to the cumulative effect of gardening participation: “...That's what this group is about: it's not just the coming together once a week for an hour...but that knock on effect of how it feels afterwards." - Kate, facilitator.

\section{Influences on well-being: The gardening group setting, tasks and group ethos}

The community gardening allotment, situated in a country field, was viewed as positively influencing well-being. Both in terms of its natural environment: "The fresh air is the best 
thing ever for myself. I just think it's fantastic..." - Jane, gardener, and through its inclusive atmosphere: "[They] help you out if you want and... If I can help someone, I'Il help someone - that would be a natural thing" - David, gardener.

An additional motif of influence concerned the tasks of gardening. These were reported by all participants to be engaging and purposeful, as well as gradable and inclusive of all levels of ability: "There might be a job to be done for which you're in pain and not able, and you might say, "Oh I'll do this but I can't do that"” - Vicky, gardener. Moreover, all gardeners noted their experience of becoming completely engaged in tasks and experiencing time distortion and a relief from pain or negative thoughts: "...you would get lost in the work, once you start a job, you just...finish it... the time goes quick alright" - Jane, gardener.

The group's person centered, member-led ethos and facilitation approach was important to participants: "It's about going back to that OT ethos of being client centered and seeing how each person develops." - William, facilitator. All participants referred to an empowering and supportive group dynamic where each member learns, and is valued, equally; "...you do need to plan it, like, but it's not dictatorial..." This approach and the focus on meaningful task-based activities were credited for promoting normalizing, easy conversation for people who usually found talking difficult: "...you just chit chat...a little bit about how they are doing... when you focus on the work someone will start telling you a bit about their mental health..." - Vicky, gardener. All facilitators and gardeners re-joined and reflected this sentiment, with one adding: "[The focus is]...not about talking. It's about the doing and the feedback from the doing..." - Kate, facilitator.

\section{Mechanisms of well-being: promoting coping, facilitating change and providing}

\section{opportunities}

\section{Promoting coping}

The gardening group was described as promoting both psychological and functional coping. In terms of psychological coping, participation in the group was viewed positively as 
a tool for dealing with life, appreciating process as well as product, and providing purpose and meaning: “... it was a bit of escapism, but it was more impassioned...It's a kind of mindfulness, even when I'm digging potatoes...It's a diversion- it's my main coping skill" Vicky, gardener.

In terms of functional coping, group engagement was cited as an organizing influence in daily life that promoted personal capacity, through the ease of planning tasks, and the tangibility of completed tasks. It was credited with supporting insight, time management and motivation to manage daily life, all seen as necessary for everyday functional coping: “...when you come home you are more motivated...I used to come home from the [gardening group] and l'd have a bit of a buzz, and be able to work, or do jobs in my own house. It's just continuous" - Jane, gardener.

\section{Facilitating change}

All participants described gardening group participation as facilitating changes in their lives by promoting interest and personal growth through growing motivation and confidence: "It gave me an interest ... Since then I have done a few courses. So, definitely, it was great" - Jane, gardener.

Garden group participation was also viewed as a way of building a more positive lifestyle: “...and you feel so much better you know, even though it was annoying me digging it, but afterwards, I felt better, and physically, I am in better shape" - David, gardener. It was also viewed as rebalancing unbalanced lifestyles through providing a sense of productivity or "work" for those who lacked it, while others perceived it as "like a holiday" or a break from everyday pressures.

\section{Providing opportunities for skill development}

All participants viewed continued engagement in the group as providing opportunities for developing their personal and professional skills. Initial development of gardening knowledge was seen as facilitating other broader skill development, such as educational, 
social, leadership skills: “... [One of the gardeners] was excellent at engaging people... in a different way from [the facilitators] and people worked better with her..." - Kate, facilitator.

\section{Discussion}

This study has found support for the positive influence of gardening group participation on well-being as defined for individuals and communities (Wilcock, 2006), has identified the role of task, setting and ethos in the effect of such group participation and has elucidated three mechanisms through which this influence occurs. These findings are in keeping with international evidence on the gardening, occupational therapy and mental health (York \& Wiseman, 2012), and begin to situate the social and therapeutic horticulture conversation in an Irish mental health context.

Findings that the natural and inclusive setting of the community garden was viewed by all participants to influence their experience of well-being reflect the work of Pretty et al (2007, 2005) and Fieldhouse (2003). The group's acknowledgement that the grading of integration (i.e. that the gardeners were supported to socialize as much as they could manage each week, depending on their ability) was a positive influence their well-being is consistent with other literature (Diamant \& Waterhouse, 2010; Wilcock, 2006) on integration and well-being in a mental health context. These findings all support occupational therapy's view of the environment as a key factor in therapy. Findings from the current study regarding the gradable and inclusive, and therefore engaging, nature of the tasks of gardening as an influence on well-being along are in synchrony with the concept of flow and engagement through meaningful and purposeful occupation (Csikszentmihalyi, 1997; Wilcock, 2006).

Findings that there was an influence through the inclusive, collaborative and member-led group's ethos and facilitation, is in line with Fieldhouse's (2003) findings regarding the cumulative and social benefits of gardening participation. They also echo views of therapists and people using mental health occupational therapy services, on how occupational therapy relationships should be conducted (Blank, 2004). 
It was found that participants viewed their well-being as being influenced through the promotion of psychological coping. These findings that coping was supported through opportunities to engage in positive and meaningful experiences, and experience motivation and a sense of agency and satisfaction in their work (Mee et al., 2004; Mee \& Sumsion 2001). Regarding functional coping, it was also observed that well-being was influenced by gardening participation in that it contributed to a sense of routine and purpose in all gardeners' days that promoted effective time use, intrinsic motivation and volition for other activities of daily living (Mee \& Sumsion, 2001).

The influence of group participation on well-being was described in terms of facilitating change, in line with findings from York and Wiseman (2012). All participants described changes they had made to their lives as a result of gardening participation. Some discussed improvements in their confidence to return to education or work; others reported increased outdoor activity and improved sense of lifestyle balance. For those with low occupational engagement, the group was seen to provide a productive role that promoted a sense of satisfaction, achievement and social interaction. For those experiencing a lack of restoration opportunities, it was described as being a source of contentment and peace that was comparable to a holiday.

All participants involved, facilitators and gardeners alike, reported a sense of well-being through skill development as a result of gardening participation. Some participants described how their gardening skills had improved and this gave them a sense of well-being and confidence to take on leadership roles in the group, and in their own lives, which in turn influenced their well-being positively. This positive influence in turn allowed them to embrace new opportunities, for some increasing their satisfaction and for others, their social contact. This self-perpetuating influence on well-being is in line with what Fieldhouse (2003) referred to as the cumulative beneficial effect of gardening participation. These mechanisms of influence are consistent with concepts by Hammell (2004) of "doing, being, becoming and 
belonging" through occupation, in the sense that gardening involves these aspects as part of its cumulative benefits: from the doing of the tasks, to the being in nature, the sense of becoming more skilled as a gardener, and the belonging of the group and the community.

The mechanisms of influence identified in this study promoting coping, facilitating change and providing opportunities for skill development, reflect findings by Casteleijn and Graham (2012) regarding domains of best practice in mental health occupational therapy services, and provide a potential framework for demonstrating the benefit of mental health occupational therapy practices.

\section{Implications of the Study}

Considering the onus on the cultural nature of well-being and its influences, this study serves to highlight the Irish context to the international conversation on the use of social and therapeutic horticulture and gardening groups in occupational therapy practice. The mechanisms of well-being reflect a westernized individual sense of well-being, however, this was situated in a community context, reflecting its importance to the participants.

The corroboration of the data gathered through interview with the data collected through ethnographic methods asserts that allotment group participation had a positive influence on gardeners' and facilitators' well-being alike. This is an addition to the supporting evidence for the use of gardening in occupational therapy practices. This research found occupational therapy-led gardening group participation to be a therapeutic medium for normalizing, supportive, satisfying, skilled group work that achieved a positive influence on well-being, as revealed by the progression of participants in their personal and professional lives. In this regard, findings create support for the role of occupational therapy in mental health services, through a focus on supporting well-being through meaningful, healthy and engaging occupations.

The facilitators' person-centered and member-led ethos was vital to the success of the group and should be encouraged for use by all therapists and students. This successful 
involvement of gardeners opinions, in the future planning and leadership of the group may be further used in practice to reflect current movements towards the human rights approaches and democratization of mental health services to people in their own communities around the world (Hammell, 2008; Patel \& Prince 2010).

\section{Limitations}

The decision was made that it would be inappropriate to collect demographic information or complete formal outcome measures for this study, as it would be contradictory to the group's non-invasive ethos. As the aim of the study was to build a case study, only a small number of participants, in one area, and specifically in one aspect of health service provision, specifically, mental health, were included. Also, the group was not facilitated exclusively by occupational therapists, leading to questions regarding the consistent emphasis on the use of gardening as a meaningful occupation versus the benefit of social support through any medium as an influence on well-being. These decisions may raise potential issues for the transferability of the study's findings.

\section{Future research}

In light of the above findings, recommendations for future research include increasing the level of participant involvement in the research and increasing the size of the study. Involving the participants consistently throughout the research process to allow their voices be heard (i.e. using a participatory action research approach) may be explored to promote meaningful change for such participants. Moreover, conducting research on the factors that make for successful gardening groups across multiple sites, on a national or international level, using multiple methods simultaneously would assist the transferability of any findings.

\section{Conclusion}

A qualitative case study with an ethnographic approach explored the influence of participation for members in a gardening group. Participants were gardeners and facilitators from a mental health service in Ireland. The emergent themes illustrate the positive 
influence of gardening group participation on well-being. This was attributed to thoughtful occupational therapy-based group facilitation, the use of meaningful, health promoting and engaging occupations in a supportive, green and knowledge-fertile environment. These factors allowed for mechanisms of influence on well-being that engaged and empowered participants to cope with life, embrace change and develop new skills for life and work, through meaningful and graded challenges.

Considerations for future gardening group use in therapeutic services may include the grading of tasks, ensuring a positive and member led ethos, and choosing an optimal setting for the garden site, both in terms of natural beauty, and ease of access. Future research involving the use of outcome measures to further capture participation in gardening groups from the gardeners and facilitators perspectives is essential. Findings are in line with current mental health occupational therapy practice, theory and research that meaningful occupations facilitate positive mental health, therefore supporting the use of gardening as a therapeutic tool for occupational therapists into the future.

Acknowledgements: The gardeners and facilitators who took part in this study, the Health Service Executive, Ireland for ethical approval, and the Clinical Therapies Department at the University of Limerick.

\section{REFERENCES}

American Horticultural Therapy Association. (2015). Horticultural Therapy

History and Practice, American Horticultural Therapy Association. Retrieved from: http://ahta.org/horticultural-therapy

Angrosino, M. (2007). Doing ethnographic and observational research. Los Angeles: Sage Publishing.

Bates, P., Gee, H., Klingel, U. \& Lippman, W. (2006). Moving towards inclusion. Mental Health Today, April, 16-18.

Blank, A. (2004). Clients Experience of Partnership with Occupational Therapists in Community Mental Health. British Journal of Occupational Therapy, 67(3), 118-124. 
Braun, V., \& Clarke, V. (2006). Using thematic analysis in psychology. Qualitative research in psychology, 3(2), 77-101.

Casteleijn, D. \& Graham, M. (2012). Domains for Occupational Therapy outcomes in mental health practices. South African Journal of Occupational Therapy, 42(1), 26-34.

Csikszentmihalyi, M. (1997). Finding flow: The psychology of engagement with everyday life. New York: Basic Books.

Diamant, E. \& Waterhouse, A. (2010). Gardening and belonging: reflections on how social and therapeutic horticulture may facilitate health, well-being and social inclusion. British Journal of Occupational Therapy, 73(2), 84-88. doi: 10.4276/030802210X12658062793924

Dictionaries, Oxford (2015). Oxford dictionaries, Oxford University Press. Retrieved from: http://www.oxforddictionaries.com/definition/english/allotment

DePoy, E. \& Gitlin, L.N. (2005). Introduction to research: Understanding and applying multiple strategies. 3rd ed. St. Louis, MO: Mosby.

Fieldhouse, J. (2003). The impact of an allotment group on client's health, well-being and social networking. British Journal of Occupational Therapy, 66(7) 286-296.

Fieldhouse, J., \& Sempik, J. (2007). 'Gardening without Borders': Reflections on the Results of a Survey of Practitioners of an 'Unstructured' Profession. British Journal of Occupational Therapy, 70(10), 449-453.

Frances, K. (2006). Outdoor recreation as an occupation to improve quality of life for people with enduring mental health problems. British Journal of Occupational Therapy, 69(4), 182-186.

Gonzalez, M.T., Hartig, T., Patil, G.G., Martinsen, E.W., \& Kirkevold, M. (2010). Therapeutic horticulture in clinical depression: a prospective study of active components. Journal of Advanced Nursing, 66(9), 2002-2013. doi: 10.1111/j.1365-2648.2010.05383

Hammell, K.W. (2008). Reflections on well-being and occupational rights. Canadian Journal of Occupational Therapy, 75(1) 61-64. doi: 10.2182/cjot.07.007

Hammell, K.W. (2004). Dimensions of meaning in the occupations of daily life. Canadian Journal of Occupational Therapy, 71(5), 296-305.

Iwama, M. (2003). Toward culturally relevant epistemologies in occupational therapy. The American journal of occupational therapy, 57(5), 582-588. 
Johnson, J. \& Schmit, H. (1983). Resource brochure for Health and Well-being. Unpublished brochure, cited in, Johnson, J. (1986). Wellness and Occupational Therapy. Australian Journal of Occupational Therapy, 40(11) 753-758.

Krefting, L. (1991). Rigor in qualitative research: the assessment of trustworthiness. American Journal of Occupational Therapy, 45(3) 214-222.

MacDougall, C. \& Fudge, E. (2001). Planning and recruiting the sample for focus groups and in-depth interviews. Qualitative health research, 11(1), 117-126.

Mason, J. (2002). Qualitative Researching. 2nd ed. Los Angeles: Sage Publishing.

Mee, J., Sumsion, T. \& Craik, C. (2004). Mental health clients confirm the value of occupation in building competence and self-identity. British Journal of Occupational Therapy, 67(5), 225-233.

Mee, J., \& Sumsion, T. (2001). Mental health clients confirm the motivating power of occupation. The British Journal of Occupational Therapy, 64(3), 121-128.

Nagle, S., Cook, V.J. \& Polatajko, H. (2002). I'm doing as much as I can: occupational choices of persons with severe and persistent mental illness. Journal of Occupational Science, 9(2) 72-81.

Newton, J. (2007). Well-being and the Natural Environment: A brief overview of the evidence. Unpublished research fellow from the Well-being in Developing Countries research group at the University of Bath. Retrieved from: http://www.savethehighlands.net/STHpdf/WellbeingAndTheNaturalEnvironmentReport.pdf

O’Connell, J.E. \& McKay, E.A. (2010). Profile, practice and perspectives of occupational therapists in community mental health teams in Ireland. British Journal of Occupational Therapy, 73(5) 218-228. doi: $10.4276 / 030802210 \times 12734991664228$

Parkinson, S., Lowe, C., \& Vecsey, T. (2011). The therapeutic benefits of horticulture in a mental health service. British Journal of Occupational Therapy, 74(11), 525-534. doi: $10.4276 / 030802211 \times 13204135680901$

Patel, V. \& Prince, M. (2010). Global mental health. Journal of the American Medical Association, 303(19), 1976-1977. doi: 10.1001/jama.2010.616

Pretty, J., Peacock, J., Hine, R., Sellens, M., South, N. \& Griffin, M. (2007). Green exercise in the UK countryside: effects on health and psychological well-being, and the implications for policy and 
planning. Journal of Environmental Planning and Management, 50(2) 211-231.

doi:10.1080/09640560601156466

Pretty, J., Peacock, J., Sellens, M. \& Griffin, M. (2005). The mental and physical health outcomes of green exercise. International Journal of Environmental Health Research, 1595, 319-337. doi: $10.1080 / 09603120500155963$

QSR International (2002). QSR NVivo2, Doncaster, Victoria, Australia: QSR International Pty. Ltd. Sempik, J., Aldridge, J. \& Becker, S. (2005). Health, well-being and social inclusion - therapeutic horticulture in the UK. Bristol: Policy Press, University of Bristol, in association with Thrive.

Sempik, J., Rickhuss, C. \& Beeston, A. (2014). The effects of social and therapeutic horticulture on aspects of social behavior. British Journal of Occupational Therapy, 77(6), 313-319. doi: $10.4276 / 030802214 X 14018723138110$

Stein, M. (2008). Community gardens for health prevention and disease prevention. International Journal for Human Caring, 12(3) 47-52.

Wilcock, A.A. (2006). An Occupational Perspective of Health. 2nd ed. New Jersey: Slack, Inc. Yin, R.K. (2009). Case study research: Design and methods. Vol.5. Los Angeles: Sage publishing. York, M. \& Wiseman, T. (2012). Gardening as an occupation: a critical review. British Journal of Occupational Therapy, 75(2), 76-84. doi: 10.4276/030802212X13286281651072. 